\title{
On the problem of detecting linear dependence for products of abelian varieties and tori
}

by

\author{
Antonella Perucca (Lausanne)
}

1. Introduction. The problem of detecting linear dependence investigates whether the property for a rational point to belong to a subgroup obeys a local-global principle.

QUESTION 1. Let $G$ be the product of an abelian variety and a torus defined over a number field $K$. Let $R$ be a point in $G(K)$ and let $\Lambda$ be a finitely generated subgroup of $G(K)$. Suppose that for all but finitely many primes $\mathfrak{p}$ of $K$ the point $(R \bmod \mathfrak{p})$ belongs to $(\Lambda \bmod \mathfrak{p})$. Does $R$ belong to $\Lambda$ ?

We answer this question affirmatively in three cases: if $\Lambda$ is cyclic; if $\Lambda$ is a free left $\operatorname{End}_{K} G$-submodule of $G(K)$; if $\Lambda$ has a set of generators (as a group) which is a basis of a free left $\operatorname{End}_{K} G$-submodule of $G(K)$. In general, we prove that there exists an integer $m$ (depending only on $G, K$ and the rank of $\Lambda$ ) such that $m R$ belongs to the left $\operatorname{End}_{K} G$-submodule of $G(K)$ generated by $\Lambda$.

The problem of detecting linear dependence for abelian varieties was first formulated by Gajda in 2002 in a letter to Ribet.

We now give the state of the art of the problem of detecting linear dependence for abelian varieties. Papers and preprints concerning this problem are: [16], 10], 2], [5], 1], 4], 7], [3], 6].

- Weston in [16] proved that if the abelian variety has commutative endomorphism ring then there exists a $K$-rational torsion point $T$ such that $R+T$ belongs to $\Lambda$. Since the torsion of the Mordell-Weil group is finite, Weston basically solved the problem for abelian varieties with commutative endomorphism ring.

2010 Mathematics Subject Classification: Primary 11G10; Secondary 14L10, 14K15.

Key words and phrases: abelian varieties, tori, reductions, local-global principles, support problem. 
- If the endomorphism ring of the abelian variety is not commutative, we are able to prove the following: there exists a non-zero integer $m$ (depending only on $G$ and $K$ ) such that $m R$ belongs to the left $\operatorname{End}_{K} G$-submodule of $G(K)$ generated by $\Lambda$; see Theorem 6 .

- We solve the problem of detecting linear dependence in the case where $\Lambda$ is a free left $\operatorname{End}_{K} G$-submodule of $G(K)$ or if $\Lambda$ has a set of generators (as a group) which is a basis of a free left $\operatorname{End}_{K} G$-submodule of $G(K)$. With an extra assumption on the point $R$ (that $R$ generates a free left $\operatorname{End}_{K} G$ submodule of $G(K)$ ), these two results are respectively proven by Gajda and Górnisiewicz in [5, Theorem B] and by Banaszak in [1, Theorem 1.1]. We remove the assumption on $R$ in Theorem 6 and in Theorem 8 respectively.

- If $\Lambda$ is cyclic, we solve the problem of detecting linear dependence. This result was only known for elliptic curves or under a condition satisfied if End $G=\mathbb{Z}$ and the dimension of $G$ is 2, 6 or odd. See [10, Theorem 3.3 and p. 120] by Kowalski.

- Gajda and Górnisiewicz in [5] use the theory of integrally semisimple Galois modules to study the problem of detecting linear dependence. This theory was completely developed by Larsen and Schoof in 111. Gajda and Górnisiewicz prove the following result ([5, Theorem A]):

Let $\ell$ be a prime such that $T_{\ell}(G)$ is integrally semisimple, let $\hat{\Lambda}$ be a free

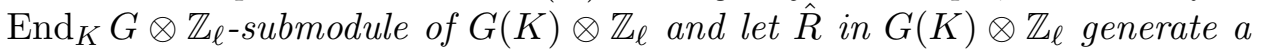
free $\operatorname{End}_{K} G \otimes \mathbb{Z}_{\ell}$-submodule of $G(K) \otimes \mathbb{Z}_{\ell}$. Then $\hat{R}$ belongs to $\hat{\Lambda}$ if and only if for all but finitely many primes $\mathfrak{p}$ of $K,(\hat{R} \bmod \mathfrak{p})$ belongs to $(\hat{\Lambda} \bmod \mathfrak{p})$. If $\operatorname{End}_{K} G \otimes \mathbb{Q}_{\ell}$ is a division algebra and $\operatorname{End}_{K} G \otimes \mathbb{Z}_{\ell}$ is a maximal order, the condition on $\hat{\Lambda}$ can be replaced by the following: $\hat{\Lambda}$ is torsion-free over $\operatorname{End}_{K} G \otimes \mathbb{Z}_{\ell}$.

Recently, new results (yet unpublished) have been proven on the problem of detecting linear dependence for abelian varieties:

- There are counterexamples. Indeed, Question 1 has a negative answer already for powers of elliptic curves. See the preprints [7] by Jossen and the author and [3] by Banaszak and Krason.

- Question 1 has an affirmative answer for simple abelian varieties. This is proven by Jossen in his thesis ([ㅌ, Corollary 8.0.2]). By the Poincaré Reducibility Theorem, an abelian variety is isogenous to $A_{1}^{e_{1}} \times \cdots \times A_{n}^{e_{n}}$, where the $A_{i}$ 's are simple and non-isogenous abelian varieties. Banaszak and Krasoń [3, Theorem A] show that there exists a $K$-rational torsion point $T$ such that $R+T$ belongs to $\Lambda$ if the following condition is satisfied: for every $i=1, \ldots, n$ the exponent $e_{i}$ is at most the dimension of $H_{1}\left(A_{i}(\mathbb{C}) ; \mathbb{Q}\right)$ as a vector space over $\operatorname{End}_{\bar{K}} A_{i} \otimes \mathbb{Q}$. Actually, $R$ belongs to $\Lambda$ because of the following result by Jossen. 
- Let $S$ be a subset of the primes of $K$ of Dirichlet density 1 . Consider the following subgroup of $G(K)$ :

$$
\tilde{\Lambda}=\{P \in G(K):(P \bmod \mathfrak{p}) \in(\Lambda \bmod \mathfrak{p}) \forall \mathfrak{p} \in S\} .
$$

This group was first studied by Kowalski in [10. Jossen [6, Theorem 8.0.1] proves (in the generality of semiabelian varieties) that the quotient $\tilde{\Lambda} / \Lambda$ is a finitely generated free abelian group.

In view of this result, Theorem 11 below can be extended to semiabelian varieties split up to isogeny. Because of Jossen's result, Theorem 6 actually proves that for semiabelian varieties split up to isogeny the following holds: the point $R$ belongs to the left $\operatorname{End}_{K} G$-submodule of $G(K)$ generated by $\Lambda$. Consequently, Question 1 has an affirmative answer whenever $\Lambda$ is a left $\operatorname{End}_{K} G$-submodule of $G(K)$. These last results are also independently proven by Jossen in [6].

Now we list further results on the problem of detecting linear dependence for commutative algebraic groups.

Schinzel [15, Theorem 2] answered Question 1 affirmatively for the multiplicative group. A generalization of Schinzel's result (Lemma 10 below for the multiplicative group where $\Lambda$ is only required to be finitely generated) was proven by Khare in [8, Proposition 3]. Question 1 has a negative answer for tori. Indeed, Schinzel [15, p. 419] gave a counterexample for the product of two copies of the multiplicative group. See Example 9 below.

Kowalski [10] studied the problem of detecting linear dependence in the case where $\Lambda$ is cyclic. In particular, he showed that the problem of detecting linear dependence has a negative answer whenever the additive group is embedded into $G$; see [10, Proposition 3.2].

Finally, a variant of the problem of detecting linear dependence was considered by Barańczuk in [4 for the multiplicative group and abelian varieties with endomorphism ring $\mathbb{Z}$.

2. Preliminaries. Let $G$ be the product of an abelian variety and a torus defined over a number field $K$. Let $R$ be a $K$-rational point of $G$ and denote by $G_{R}$ the smallest algebraic $K$-subgroup of $G$ containing $R$. Write $G_{R}^{0}$ for the connected component of the identity of $G_{R}$ and write $n_{R}$ for the number of connected components of $G_{R}$. By [13, Proposition 5], $G_{R}^{0}$ is the product of an abelian variety and a torus defined over $K$.

We say that $R$ is independent if $R$ is non-zero and $G_{R}=G$. The point $R$ is independent in $G$ if and only if $R$ is independent in $G \times{ }_{K} \bar{K}$. Furthermore, $R$ is independent in $G$ if and only if $R$ is non-zero and the left $\operatorname{End}_{K} G$ submodule of $G(K)$ generated by $R$ is free. See [13, Section 2]. 
Lemma 2. Let $R$ be a $K$-rational point of $G$ and let $d$ be a non-zero integer. We have $G_{d R}^{0}=G_{R}^{0}$. In particular, the dimension of $G_{d R}$ equals the dimension of $G_{R}$ and $G_{n_{R} R}=G_{n_{R} R}^{0}=G_{R}^{0}$.

Proof. Since $G_{R}$ contains $d R$ we have $G_{d R} \subseteq G_{R}$ and so $G_{d R}^{0} \subseteq G_{R}^{0}$. Hence it suffices to prove that $G_{d R}^{0}$ and $G_{R}^{0}$ have the same dimension. Clearly, the dimension of $G_{d R}^{0}$ is less than or equal to the dimension of $G_{R}^{0}$. To prove the other inequality it suffices to show that multiplication by $[d]$ maps $G_{R}$ into $G_{d R}$. This is true because $[d]^{-1} G_{d R}$ contains $R$.

Denote by $W$ the connected component of $G_{R}$ containing $R$ and let $X$ be a torsion point in $G_{R}(\bar{K})$ such that $W=X+G_{R}^{0}$ (see [13, Lemma 1]). Clearly, $n_{R} X$ is the least positive multiple of $X$ belonging to $G_{R}^{0}$ and the connected components of $G_{R}$ are of the form $a X+G_{R}^{0}$ for $0 \leq a<n_{R}$. We can write $R=X+Z$ where $Z$ is in $G_{R}^{0}(\bar{K})$. Since $R$ and $Z$ have a common multiple, from Lemma 2 it follows that $Z$ is independent in $G_{R}^{0}$.

Lemma 3. Let $L$ be a finite extension of $K$ where $X$ is defined. Then for all but finitely many primes $\mathfrak{q}$ of $L$ the point $\left(n_{R} X \bmod \mathfrak{q}\right)$ is the least positive multiple of $(X \bmod \mathfrak{q})$ belonging to $\left(G_{R}^{0} \bmod \mathfrak{q}\right)$.

Proof. Denote by $x$ the order of $X$. We may assume that the points in $G_{R}[x]$ are defined over $L$. Suppose that $d$ is a positive integer smaller than $n_{R}$ such that for infinitely many primes $\mathfrak{q}$ of $L$ the point $(d X \bmod \mathfrak{p})$ belongs to $\left(G_{R}^{0} \bmod \mathfrak{q}\right)$. Up to excluding finitely many primes $\mathfrak{q}$, we may assume that the reduction modulo $\mathfrak{q}$ maps injectively $G_{R}[x]$ to $\left(G_{R} \bmod \mathfrak{q}\right)[x]$. By [10. Lemma 4.4] we may also assume that the reduction modulo $\mathfrak{q}$ maps surjectively $G_{R}^{0}[x]$ onto $\left(G_{R}^{0} \bmod \mathfrak{q}\right)[x]$. Then for infinitely many primes $\mathfrak{q}$ the point $(d X \bmod \mathfrak{q})$ belongs to the reduction modulo $\mathfrak{q}$ of the finite group $G_{R}^{0}[x]$. We deduce that $d X$ belongs to $G_{R}^{0}[x]$. We have a contradiction since $n_{R} X$ is the least positive multiple of $X$ which belongs to $G_{R}^{0}$.

Lemma 4. Let $A$ and $T$ be respectively an abelian variety and a torus defined over a number field $K$. Then $\operatorname{Hom}_{\bar{K}}(A, T)=\{0\}$ and $\operatorname{Hom}_{\bar{K}}(T, A)$ $=\{0\}$.

Proof. Since $A$ is a complete variety and $T$ is affine, there are no nontrivial morphisms from $A$ to $T$. To prove the other equality, suppose that $\phi$ is a morphism from $\mathbb{G}_{m}$ to $A$. On the point sets, $\phi$ gives a homomorphism from a non-finitely generated to a finitely generated abelian group. Then the kernel of $\phi$ is not finite so it must be the whole $\mathbb{G}_{m}$.

The following lemma in the case of abelian varieties was proven by Banaszak in [1, Step 2 of the proof of Theorem 1.1].

Lemma 5. Let $G$ be the product of an abelian variety and a torus defined over a number field $K$. Let $\alpha$ be a $\bar{K}$-endomorphism of $G$. Suppose that there 
exists a prime number $\ell$ such that for every $n>0$ and every torsion point $T$ of $G$ of order $\ell^{n}$ the point $\alpha(T)$ is a multiple of $T$. Then $\alpha$ is a scalar.

Proof. Let $R$ be a commutative ring with 1 . Let $F$ be a free finitely generated $R$-module. Suppose that $s$ is an $R$-endomorphism of $F$ sending every element to a multiple of itself. Then it can easily be seen that $s$ is a scalar. Apply the previous assertion to $R=\mathbb{Z} / \ell^{n} \mathbb{Z}, F=G\left[\ell^{n}\right]$, taking for $s$ the image of $\alpha$ in $\operatorname{End}_{\mathbb{Z}} G\left[\ell^{n}\right]$. We deduce that $\alpha$ acts as a scalar on $G\left[\ell^{n}\right]$. So for every $n>0$ there exists an integer $c_{n}$ such that $\alpha$ acts as the multiplication by $c_{n}\left(\bmod \ell^{n}\right)$ on $G\left[\ell^{n}\right]$. Since $\alpha$ commutes with multiplication by $\ell$ we deduce that $c_{n+1} \equiv c_{n}\left(\bmod \ell^{n}\right)$ for every $n$. This means that there exists $c$ in $\mathbb{Z}_{\ell}$ such that $c \equiv c_{n}\left(\bmod \ell^{n}\right)$ for every $n$ and that $\alpha$ acts on $T_{\ell} G$ as the multiplication by $c$.

Write $G=A \times T$ where $A$ is an abelian variety and $T$ is a torus. By Lemma 4, $\alpha$ is the product $\alpha_{A} \times \alpha_{T}$ of an endomorphism of $A$ and an endomorphism of $T$. It suffices to prove the following: if $A$ (respectively $T$ ) is non-zero then $c$ is an integer and $\alpha_{A}$ (respectively $\alpha_{T}$ ) is the multiplication by $c$.

Suppose that $A$ is non-zero. We know that $\alpha_{A}$ acts on $T_{\ell} A$ as the multiplication by $c$. By [12, Theorem 3, p. 176], $c$ is an integer and $\alpha_{A}$ is the multiplication by $c$.

Suppose that $T$ is non-zero. We reduce at once to the case where $T=\mathbb{G}_{m}^{h}$ for some $h \geq 1$. The endomorphism ring of $\mathbb{G}_{m}$ is $\mathbb{Z}$ hence we can identify the endomorphism ring of $T$ with the ring of $h \times h$-matrices with integer coefficients. Since $\alpha_{T}$ acts on $T_{\ell} T$ as the multiplication by $c$, we deduce that $\alpha_{T}$ is a scalar matrix. Hence $c$ is an integer and $\alpha_{T}$ is the multiplication by $c$.

3. On a result by Gajda and Górnisiewicz. In this section we apply results on the support problem ([14]) to study the problem of detecting linear dependence. The second assertion of the following theorem was proven by Gajda and Górnisiewicz in [5, Theorem B] under the assumption that the point $R$ generates a free left $\operatorname{End}_{K} G$-submodule of $G(K)$.

THeOREM 6 . Let $G$ be the product of an abelian variety and a torus defined over a number field $K$. Let $R$ be a $K$-rational point of $G$ and let $\Lambda$ be a finitely generated subgroup of $G(K)$. Suppose that for all but finitely many primes $\mathfrak{p}$ of $K$ the point $(R \bmod \mathfrak{p})$ belongs to $(\Lambda \bmod \mathfrak{p})$. Then there exists a non-zero integer $m$ (depending only on $G, K$ and the rank of $\Lambda$ ) such that $m R$ belongs to the left $\operatorname{End}_{K} G$-submodule of $G(K)$ generated by $\Lambda$. Furthermore, if $\Lambda$ is a free left $\operatorname{End}_{K} G$-submodule of $G(K)$ then $R$ belongs to $\Lambda$.

Remark that if $G$ is an abelian variety, the integer $m$ in Theorem 6 depends only on $G$ and $K$ since the rank of $\Lambda$ is bounded by the rank of the Mordell-Weil group. 
Lemma 7. Let $G$ be the product of an abelian variety and a torus defined over a number field $K$. Let $R$ be a $K$-rational point of $G$ and let $\Lambda$ be a finitely generated subgroup of $G(K)$. Fix a rational prime $\ell$. Suppose that for all but finitely many primes $\mathfrak{p}$ of $K$ there exists an integer $c_{\mathfrak{p}}$ coprime to $\ell$ such that $\left(c_{\mathfrak{p}} R \bmod \mathfrak{p}\right)$ belongs to $(\Lambda \bmod \mathfrak{p})$. Then there exists a non-zero integer $c$ such that $c R$ belongs to the left $\operatorname{End}_{K} G$-submodule of $G(K)$ generated by $\Lambda$. One can take $c$ such that $v_{\ell}(c) \leq v_{\ell}(m)$ where $m$ is a non-zero integer depending only on $G, K$ and the rank of $\Lambda$ (hence not depending on $\ell$ ). If $\Lambda$ is a free left $\operatorname{End}_{K} G$-submodule of $G(K)$, one can take $m=1$.

Proof. Let $P_{1}, \ldots, P_{s}$ generate $\Lambda$ as a $\mathbb{Z}$-module. Consider $G^{s}$ and its $K$ rational points $P=\left(P_{1}, \ldots, P_{s}\right)$ and $Q=(R, 0, \ldots, 0)$. We can apply [14, Main Theorem] to the points $P$ and $Q$. Then there exist a $K$-endomorphism $\phi$ of $G^{s}$ and a non-zero integer $c$ such that $\phi(P)=c Q$. By [14, Proposition 10] one can take $c$ such that $v_{\ell}(c) \leq v_{\ell}(m)$ where $m$ depends only on $G^{s}$ and $K$. In particular, $c R$ belongs to $\operatorname{End}_{K} G \cdot \Lambda$. Since $s$ depends only on $G, K$ and the rank of $\Lambda$, the first assertion is proven. For the second assertion, let $P_{1}, \ldots, P_{s}$ be a basis of $\Lambda$ as a left $\operatorname{End}_{K} G$-module. Since $P$ is independent, by [14, Proposition 9] one can take $c$ coprime to $\ell$. Consequently, one can take $m=1$.

Proof of Theorem 6. We apply Lemma 7 to every rational prime $\ell$. Then for every $\ell$ there exists an integer $c_{\ell}$ such that $c_{\ell} R$ belongs to $\operatorname{End}_{K} G \cdot \Lambda$ and $v_{\ell}\left(c_{\ell}\right) \leq v_{\ell}(m)$, where $m$ is a non-zero integer depending only on $G, K$ and the rank of $\Lambda$. Since $m$ is in the ideal of $\mathbb{Z}$ generated by the $c_{\ell}$ 's, we deduce that $m R$ belongs to $\operatorname{End}_{K} G \cdot \Lambda$. If $\Lambda$ is a free left $\operatorname{End}_{K} G$-submodule of $G(K)$, one can take $m=1$ in Lemma 7 , hence $R$ belongs to $\Lambda$.

4. A refinement of a result by Banaszak. In this section we extend the result by Banaszak on the problem of detecting linear dependence ([1, Theorem 1.1]) from abelian varieties to products of abelian varieties and tori. Furthermore, by adapting Banaszak's proof we are able to remove his assumption on the point $R$ (that $R$ generates a free left $\operatorname{End}_{K} G$-submodule of $G(K)$ ).

THEOREM 8. Let $G$ be the product of an abelian variety and a torus defined over a number field $K$. Let $\Lambda$ be a finitely generated subgroup of $G(K)$ such that it has a set of generators (as a group) which is a basis of a free left $\operatorname{End}_{K} G$-submodule of $G(K)$. Let $R$ be a point in $G(K)$. Suppose that for all but finitely many primes $\mathfrak{p}$ of $K$ the point $(R \bmod \mathfrak{p})$ belongs to $(\Lambda \bmod \mathfrak{p})$. Then $R$ belongs to $\Lambda$.

If $\operatorname{End}_{K} G=\mathbb{Z}$, the assumption on $\Lambda$ is equivalent to saying that $\Lambda$ contains no torsion points. In general, the condition implies that the left 
End $_{K} G$-module generated by $\Lambda$ is free. The following example by Schinzel shows that the latter assumption is not sufficient.

Example 9 (Schinzel, [15, p. 419]). A counterexample to Question 1 for $G=\mathbb{G}_{m}^{2}$ and $K=\mathbb{Q}$ is the following. Take the point $R=(1,4)$ and take the group $\Lambda$ generated by the points $P_{1}=(2,1), P_{2}=(3,2), P_{3}=(1,3)$. For every prime number $\mathfrak{p}$ the point $(R \bmod \mathfrak{p})$ belongs to $(\Lambda \bmod \mathfrak{p})$. The point $R$ belongs to the left $\operatorname{End}_{K} G$-module generated by $\Lambda$ but does not belong to $\Lambda$. Notice that the left $\operatorname{End}_{K} G$-module generated by $\Lambda$ is free and it is generated by $P_{2}$.

Lemma 10. Let $G$ be the product of an abelian variety and a torus defined over a number field $K$. Let $\Lambda$ be a finitely generated subgroup of $G(K)$ such that it has a set of generators (as a group) which is a basis of a free left $\operatorname{End}_{K} G$-submodule of $G(K)$. Let $R$ be a point in $G(K)$. Fix a prime number $\ell$. Suppose that for all but finitely many primes $\mathfrak{p}$ of $K$ there exists an integer $c_{\mathfrak{p}}$ coprime to $\ell$ such that the point $\left(c_{\mathfrak{p}} R \bmod \mathfrak{p}\right)$ belongs to $(\Lambda \bmod \mathfrak{p})$. Then there exists an integer $c$ coprime to $\ell$ such that $c R$ belongs to $\Lambda$.

Proof. By Lemma 7 applied to $\operatorname{End}_{K} G \cdot \Lambda$, there exists an integer $c$ coprime to $\ell$ such that $c R$ belongs to $\operatorname{End}_{K} G \cdot \Lambda$. Let $\left\{P_{1}, \ldots, P_{n}\right\}$ be a set of generators for $\Lambda$ which is a basis for $\operatorname{End}_{K} G \cdot \Lambda$. We can write

$$
c R=\sum_{i=1}^{n} \phi_{i} P_{i}
$$

for some $\phi_{i}$ in $\operatorname{End}_{K} G$. Without loss of generality it suffices to prove that $\phi_{1}$ is the multiplication by an integer.

Suppose that $\phi_{1}$ is not multiplication by an integer and apply Lemma 5 to $\phi_{1}$. Then there exists a point $T$ in $G\left[\ell^{\infty}\right]$ such that $\phi_{1}(T)$ is not a multiple of $T$. Let $L$ be a finite extension of $K$ where $T$ is defined. The point $\left(P_{1}-T\right.$, $\left.P_{2}, \ldots, P_{n}\right)$ is independent in $G^{n}$ hence by [13, Proposition 12] there are infinitely many primes $\mathfrak{q}$ of $L$ such that the following holds: $\left(P_{i} \bmod \mathfrak{q}\right)$ has order coprime to $\ell$ for every $i \neq 1$ and $\left(P_{1}-T \bmod \mathfrak{q}\right)$ has order coprime to $\ell$. By discarding finitely many primes $\mathfrak{q}$, we may assume the following: the order of $(T \bmod \mathfrak{q})$ equals the order of $T$; the point $\left(\phi_{1}(T) \bmod \mathfrak{q}\right)$ is not a multiple of $(T \bmod \mathfrak{q})$ and in particular it is non-zero; $\left(c_{\mathfrak{q}} R \bmod \mathfrak{q}\right)$ belongs to $(\Lambda \bmod \mathfrak{q})$ for some integer $c_{\mathfrak{q}}$ coprime to $\ell$.

Fix $\mathfrak{q}$ as above. We know that there exists an integer $m$ coprime to $\ell$ such that $\left(m P_{i} \bmod \mathfrak{q}\right)=0$ for every $i \neq 1$ and $\left(m\left(P_{1}-T\right) \bmod \mathfrak{q}\right)=0$. Then we have

$$
\left(m c_{\mathfrak{q}} c R \bmod \mathfrak{q}\right)=\left(m c_{\mathfrak{q}} \phi_{1}\left(P_{1}\right) \bmod \mathfrak{q}\right)=\left(m c_{\mathfrak{q}} \phi_{1}(T) \bmod \mathfrak{q}\right) .
$$

Since $v_{\ell}\left(m c_{\mathfrak{q}}\right)=0$, we deduce that the point $\left(m c_{\mathfrak{q}} c R \bmod \mathfrak{q}\right)$ has order a 
power of $\ell$ and it is not a multiple of $(T \bmod \mathfrak{q})$. Then $\left(m c_{\mathfrak{q}} c R \bmod \mathfrak{q}\right)$ does not belong to $\sum_{i=1}^{n} \mathbb{Z}\left(P_{i} \bmod \mathfrak{q}\right)$. Consequently, $\left(c_{\mathfrak{q}} R \bmod \mathfrak{q}\right)$ does not belong to $(\Lambda \bmod \mathfrak{q})$ and we have a contradiction.

Proof of Theorem 8. We can apply Lemma 10 to every rational prime $\ell$. Then for every $\ell$ there exists an integer $c_{\ell}$ coprime to $\ell$ such that $c_{\ell} R$ belongs to $\Lambda$. Since 1 is contained in the ideal of $\mathbb{Z}$ generated by the $c_{\ell}$ 's, we deduce that $R$ belongs to $\Lambda$.

5. On a result by Kowalski. Kowalski [10] studied the problem of detecting linear dependence for commutative algebraic groups in the case where $\Lambda$ is cyclic. The following theorem was proven for elliptic curves in [10, Theorem 3.3]. Kowalski also described in [10, p. 120] how to apply the results by Khare and Prasad [9] to this problem.

THEOREM 11. Let $G$ be the product of an abelian variety and a torus defined over a number field $K$. Let $\Lambda$ be a cyclic subgroup of $G(K)$. Let $R$ be a $K$-rational point of $G$. Suppose that for all but finitely many primes $\mathfrak{p}$ of $K$ the point $(R \bmod \mathfrak{p})$ belongs to $(\Lambda \bmod \mathfrak{p})$. Then $R$ belongs to $\Lambda$.

Lemma 12. Let $G$ be the product of an abelian variety and a torus defined over a number field $K$. Let $\Lambda$ be an infinite cyclic subgroup of $G(K)$. Let $T$ be a K-rational torsion point of $G$. Suppose that for all but finitely many primes $\mathfrak{p}$ of $K$ the point $(T \bmod \mathfrak{p})$ belongs to $(\Lambda \bmod \mathfrak{p})$. Then $T$ is zero.

Proof. Suppose that $T$ is non-zero. Then $T$ can be uniquely written as a sum of torsion points whose orders are prime powers. These torsion points are multiples of $T$. Consequently, we reduce at once to the case where the order of $T$ is the power of a prime number $\ell$.

Let $\Lambda=\mathbb{Z} P$ for a point $P$ of infinite order. The algebraic subgroup $G_{P}$ of $G$ generated by $P$ has dimension at least 1 . In Section 2 we saw the following: $P=X+Z$ for some point $Z$ in $G_{P}^{0}(\bar{K})$ and some torsion point $X$ in $G_{P}(\bar{K})$; the point $Z$ is independent in $G_{P}^{0} ; n_{P} X$ is the least multiple of $X$ which belongs to $G_{P}^{0} ; G_{P}^{0}$ is the product of an abelian variety and a torus defined over $K$.

Let $c$ be the $\ell$-adic valuation of the order of $X$. Let $L$ be a finite extension of $K$ where $X, Z, G\left[\ell^{2 c}\right]$ are defined and such that $n_{P} X$ has $n_{P}$-roots in $G_{P}^{0}(L)$. Notice that for all but finitely many primes $\mathfrak{q}$ of $L$ the point $(T \bmod \mathfrak{q})$ belongs to $(\mathbb{Z} P \bmod \mathfrak{q})$.

By [13, Proposition 12], there exist infinitely many primes $\mathfrak{q}$ of $L$ such that the order of $(Z \bmod \mathfrak{q})$ is coprime to $\ell$. Then for infinitely many primes $\mathfrak{q}$ the point $(T \bmod \mathfrak{q})$ lies in the finite group generated by $(X \bmod \mathfrak{q})$. We deduce that $T=a X$ for some non-zero integer $a$. 
Let $T_{0}$ be a point in $G_{P}^{0}$ of order $\ell^{2 c}$. By [13, Proposition 11], there exist infinitely many primes $\mathfrak{q}$ of $L$ such that the order of $\left(Z-T_{0} \bmod \mathfrak{q}\right)$ is coprime to $\ell$. We deduce that for infinitely many primes $\mathfrak{q}$ the point $(T \bmod \mathfrak{q})$ lies in the finite group generated by $\left(T_{0}+X \bmod \mathfrak{q}\right)$. Then $T=b\left(T_{0}+X\right)$ for some non-zero integer $b$.

Since $a X=b\left(T_{0}+X\right)$ and because the order of $T_{0}$ is $\ell^{2 c}$ we deduce that $v_{\ell}(b) \geq c$. Consequently, $T$ is the sum of $b T_{0}$ and a torsion point of order coprime to $\ell$. Then $T$ is a multiple of $T_{0}$ and in particular it belongs to $G_{P}^{0}$.

Let $T_{1}$ be a point in $G_{P}^{0}(L)$ such that $n_{P} T_{1}=-n_{P} X$. By [13, Proposition 11], there exist infinitely many primes $\mathfrak{q}$ of $L$ such that the order of $\left(Z-T_{1} \bmod \mathfrak{q}\right)$ is coprime to $\ell$. Up to discarding finitely many primes $\mathfrak{q}$, we may assume that $(T \bmod \mathfrak{q})$ belongs to $(\mathbb{Z} P \bmod \mathfrak{q})$ and that the order of $(T \bmod \mathfrak{q})$ equals the order of $T$. Again up to discarding finitely many primes $\mathfrak{q}$, by Lemma 3 we may assume that $\left(n_{P} X \bmod \mathfrak{q}\right)$ is the least multiple of $(X \bmod \mathfrak{q})$ belonging to $\left(G_{P}^{0} \bmod \mathfrak{q}\right)$. Consequently, the intersection of $\left(G_{P}^{0} \bmod \mathfrak{q}\right)$ and $(\mathbb{Z} P \bmod \mathfrak{q})$ is $\left(\mathbb{Z} n_{P} P \bmod \mathfrak{q}\right)$. Then $(T \bmod \mathfrak{q})$ belongs to $\left(\mathbb{Z} n_{P} P \bmod \mathfrak{q}\right)$.

Fix a prime $\mathfrak{q}$ as above and denote by $r$ the order of $\left(Z-T_{1} \bmod \mathfrak{q}\right)$. We have

$$
\begin{aligned}
\left(r n_{P} P \bmod \mathfrak{q}\right) & =\left(r n_{P} Z+r n_{P} X \bmod \mathfrak{q}\right)=\left(r n_{P} T_{1}+r n_{P} X \bmod \mathfrak{q}\right) \\
& =(0 \bmod \mathfrak{q}) .
\end{aligned}
$$

Since $r$ is coprime to $\ell$, it follows that $\left(\mathbb{Z} n_{P} P \bmod \mathfrak{q}\right)$ has no $\ell$-torsion and in particular it does not contain $(T \bmod \mathfrak{q})$. We have a contradiction.

Proof of Theorem 11. If $\Lambda$ is finite then there exists an element $P^{\prime}$ in $\Lambda$ such that for infinitely many primes $\mathfrak{p}$ of $K$ we have $(R \bmod \mathfrak{p})=\left(P^{\prime} \bmod \mathfrak{p}\right)$. Hence $R=P^{\prime}$ and the statement is proven. We may then assume that $\Lambda=\mathbb{Z} P$ for a point $P$ of infinite order.

We first prove that the statement holds in the case where the algebraic group $G_{P}$ generated by $P$ is connected. In this case, $G_{P}$ is the product of an abelian variety and a torus ([13, Proposition 5]). By [10, Lemma 4.2], we may assume that $G_{P}=G$. So we may assume that $P$ is independent in $G$ and we conclude by applying Theorem 8 .

In general, let $n_{P}$ be the number of connected components of $G_{P}$. Notice that the points $n_{P} P$ and $n_{P} R$ still satisfy the hypotheses of the theorem and that $G_{n_{P} P}$ is connected by Lemma 2 . Therefore we know (by the special case above) that $n_{P} R=g n_{P} P$ for some integer $g$. Since $R$ and $P$ are rational points, we deduce that $R=g P+T$ for some rational torsion point $T$. Since $R-T$ belongs to $\Lambda$, for all but finitely many primes $\mathfrak{p}$ of $K$ the point $(T \bmod \mathfrak{p})$ belongs to $(\Lambda \bmod \mathfrak{p})$. By applying Lemma 12 we deduce that $T=0$ hence $R$ belongs to $\Lambda$. 
Acknowledgements. I thank Emmanuel Kowalski and René Schoof for helpful discussions.

\section{References}

[1] G. Banaszak, On a Hasse principle for Mordell-Weil groups, C. R. Math. Acad. Sci. Paris 347 (2009), 709-714.

[2] G. Banaszak, W. Gajda, and P. Krasoń, Detecting linear dependence by reduction maps, J. Number Theory 115 (2005), 322-342.

[3] G. Banaszak and P. Krason, On arithmetic in Mordell-Weil groups, preprint, arXiv: 0904.2848v2, 2009.

[4] S. Barańczuk, On a generalization of the support problem of Erdös and its analogues for abelian varieties and K-theory, J. Pure Appl. Algebra 214 (2010), 380-384.

[5] W. Gajda and K. Górnisiewicz, Linear dependence in Mordell-Weil groups, J. Reine Angew. Math. 630 (2009), 219-233.

[6] P. Jossen, On the arithmetic of 1-motives, Ph.D. thesis, Central European Univ. Budapest, 2009.

[7] P. Jossen and A. Perucca, A counterexample to the local-global principle of linear dependence for abelian varieties, C. R. Math. Acad. Sci. Paris 348 (2010), 9-10.

[8] C. Khare, Compatible systems of mod p Galois representations and Hecke characters, Math. Res. Lett. 10 (2003), 71-83.

[9] C. Khare and D. Prasad, Reduction of homomorphisms mod $p$ and algebraicity, J. Number Theory 105 (2004), 322-332.

[10] E. Kowalski, Some local-global applications of Kummer theory, Manuscripta Math. 111 (2003), 105-139.

[11] M. Larsen and R. Schoof, Whitehead's lemma and Galois cohomology of abelian varieties, http://mlarsen.math.indiana.edu/ larsen/unpublished.html, 2004.

[12] D. Mumford, Abelian Varieties, Tata Inst. Fund. Res. Stud. Math. 5, Oxford Univ. Press, London, 1970.

[13] A. Perucca, Prescribing valuations of the order of a point in the reductions of abelian varieties and tori, J. Number Theory 129 (2009), 469-476.

[14] - , Two variants of the support problem for products of abelian varieties and tori, ibid., 1883-1892.

[15] A. Schinzel, On power residues and exponential congruences, Acta Arith. 27 (1975), 397-420.

[16] T. Weston, Kummer theory of abelian varieties and reductions of Mordell-Weil groups, ibid. 110 (2003), 77-88.

Antonella Perucca

Section de Mathématiques

École Polytechnique Fédérale de Lausanne

Station 8

CH-1015 Lausanne, Switzerland

E-mail: antonella.perucca@epfl.ch

Received on 14.11.2008

and in revised form on 19.8.2009 